Vol 11, Issue 10, 2018

\title{
IMPACT OF GLAUCOMA IN COGNITIVE DECLINE
}

\author{
ARIJITA BANERJEE ${ }^{1}$, INDU KHURANA ${ }^{2}$
}

${ }^{1}$ Department of Physiology, Maulana Azad Medical College, New Delhi, India. ${ }^{2}$ Department of Physiology, World College of Medical Sciences and Research, Haryana, India. Email: b.arijita@gmail.com

Received: 02 May 2018, Revised and Accepted: 28 June 2018

\section{ABSTRACT}

Objective: The objective of the study was to assess cognitive performance differences, if any, among primary open-angle glaucoma (POAG) patients, normal-tension glaucoma (NTG) patients, and healthy control (C) subjects.

Methods: 60 age and sex-matched subjects (20 POAG, 20 NTG, and 20 C subjects) were included in this study. Following routine ophthalmological examination the cognitive performance of all participants, detailed neurological examinations, including the mini-mental state examination (MMSE), were done.

Results: The mean retinal nerve fiber layer thicknesses were significantly different among the groups in the POAG, NTG, and C subjects, respectively $(\mathrm{p}<0.001)$. MMSE scores were 24.1 $\pm 2.4,25.2 \pm 3.8$, and 28.9 \pm 0.9 in the POAG, NTG, and controls groups, respectively. There were significant differences among the three groups $(\mathrm{p}<0.001)$. Moreover, there were significant differences between the NTG and controls groups ( $<<0.001)$, and between the POAG and controls groups $(\mathrm{p}<0.001)$.

Conclusions: The overlapping pathogenesis of glaucoma and neurodegenerative disorders cause deterioration in cognitive performance. Decreased values of MMSE scores in POAG and NTG patients compared to the scores of healthy controls suggest a detailed neurological examination of glaucoma patients.

Keywords: Neurodegenerative disease, Cognition, Dementia, Open-angle glaucoma.

(C) 2018 The Authors. Published by Innovare Academic Sciences Pvt Ltd. This is an open access article under the CC BY license (http://creativecommons. org/licenses/by/4. 0/) DOI: http://dx.doi.org/10.22159/ajpcr.2018.v11i10.27068

\section{INTRODUCTION}

Glaucoma, the second chronic cause of blindness in the world, is featured by a gradual loss of retinal ganglion cells (RGCs), atrophy of optic nerve and visual field defects. Primary open angle glaucoma (POAG) forms the main area of research nowadays where the important risk factor is elevated intraocular pressure (IOP). Another subset of open-angle glaucoma is normal tension glaucoma (NTG) in which there is optic nerve degeneration without elevation of IOP [1].

With a growing old population, people are at more risk of visual loss caused by aging process and dementia. Dementia is a gradual neurodegenerative disease featured by cognitive dysfunction, memory impairment, personality changes, and behavioral disturbances. Alzheimer dementia (AD) may be more frequent among glaucoma patients which are attributed to common genetic risk factors and similar pathological changes in the optic nerves. A study has shown the causal relationship of decreased cerebrospinal fluid pressure (CSFP) in patients with the AD. The results by Mandas et al. showed that elevated IOP, reduced CSFP, or both could lead to an increased translaminar pressure difference, following glaucomatous optic nerve damage $[2,3]$.

There are few studies showing associations between age-related degenerative eye diseases including cataract, glaucoma, age-related macular degeneration (AMD), and dementia. Only a few studies analyzed the potential link between glaucoma and cognitive function impairment and found controversial results $[4,5]$.

Till now, the mini-mental state examination (MMSE) is the best-studied method of analyzing cognitive impairment. This test is scored of 30, a score of 24 or less suggest dementia [6,7].
With the above tools of cognitive assessment, the aim of our study was to determine if there are differences among the POAG, NTG, and healthy controls in terms of cognitive skills.

\section{METHODS}

The research followed the tenets of the Declaration of Helsinki and consent of the Ethical Committee was obtained for the study protocol. All patients provided written informed consent before participation. The study sample comprised three groups between age 45 and 65 years: Group I - 20 age and sex-matched healthy controls, Groups II - 20 patients with NTG, and Group III - 20 patients with POAG.

Inclusion criteria for Group III (POAG) are: IOP $>21 \mathrm{mmHg}$ without treatment, optic disc changes with neuro-retinal rim notching, optic disc excavation, vertical or horizontal cup to disk (C/D) ratio $>0.5$ or C/D asymmetry between 2 eyes $>0.2$, peripapillary splinter hemorrhages, visual field outside normal limits on Humphrey automated perimetry on three perimetry readings, and all angles $\left(360^{\circ}\right)$ open on gonioscopy.

Inclusion criteria for Group II (NTG) are same as for POAG except IOP ranging between 10 and $21 \mathrm{mmHg}$.

Exclusion criteria include patients with AMD, secondary causes of glaucoma, hazy media, optic neuritis, any disease involving the macula, retina, or visual pathway, high myopia ( $>6$ dioptre), previous intraocular surgery and on drugs known to cause optic neuropathy, and patients with diabetes mellitus and hypertension.

All subjects in this study underwent an ophthalmological examination including visual acuity with Snellen chart, IOP measurement using Goldmann's applanation tonometer, measurement of central corneal thickness (CCT) by an optical pachymetry, gonioscopy, and fundus 
examination for glaucoma diagnosis and grouping. Neurological examination including motor, sensory and cerebellar system examination was done in all the subjects following which the concerned investigation of our research that is a cognitive function for scanning of dementia in these subjects was carried out using MMSE.

In MMSE orientation, attention, memory, language, and shape copying are assessed. The maximum score is 30 , and the cutoff value as was determined by Gungen and his associates in 2002 to be 24 [8]. Longterm memory, short-term memory, attention span, calculation, naming of items, performance of task in three steps, reading, writing, and assembling abilities were all measured in MMSE. A little training is required before the execution of the test, and it usually takes about $10 \mathrm{~min}$ and has vast medical acceptance. In clinical practice, a rough rule of thumb is practiced that patients with mild dementia generally seen having a score of 18-26 of 30, while those with moderate dementia a score of 10-18, and subjects with severe dementia a score of $<10$. At scores above the level of 24 , the MMSE lacks sensitivity for the diagnosis of mild dementia, and other assessments are needed. The MMSE focuses on memory, attention, construction, and orientation domains.

The statistical data analysis was performed using software SPSS Version 20.0 (SPSS, Inc., IBM Corp., USA). All variables were defined by methods of descriptive statistics. The analysis of the quantitative variables included calculation of the mean and standard deviation $(\times[S D])$. One-way analysis of variance was used to compare the differences among the three groups for each variable. Pairwise comparison of the MMSE scores between the controls and NTG and POAG patients were done using Mann-Whitney U-test. $\mathrm{p}<0.05$ was regarded as significant statistically.

\section{RESULTS}

The mean age values of Group I (controls), Group II (NTG), and Group III (POAG) were $51.6 \pm 5.25,53.45 \pm 4.88$, and $51.75 \pm 6.07$ years, respectively. There were no significant differences with respect to age among the groups ( $\mathrm{p}=0.32$ ). Of 20 NTG patients, $55 \%$ were males and $45 \%$ were females, in POAG patients 65\% were males and 35\% were females, whereas in control group $60 \%$ were males and $40 \%$ were females. The mean basal value of IOP in Groups I and II was within normal range and comparable; however, the mean basal IOP was significantly higher $(26.3 \pm 1.75)$ in Group III $(\mathrm{p}<0.000)$.

Table 1 shows significant statistical differences in CCT among the three groups. While comparing the groups pairwise, we found the statistically significant difference between controls and NTG $(p=0.004)$ along with a high degree of significant difference between controls and POAG $(\mathrm{p}<0.001)$. The mean retinal nerve fiber layer (RNFL) thickness measurements among three groups also found to be statistically significant. Further on comparing GroupWise significant differences were found between NTG and POAG ( $p=0.021)$ and the NTG and control groups $(\mathrm{p}<0.002)$

The MMSE scores, when compared together, the differences among the three groups were statistically significant $(p<0.001)$. On comparing the scores GroupWise individually, we could find a significant difference between controls and NTG $(\mathrm{p}<0.001)$ and between controls and POAG $(\mathrm{p}<0.001)$, with no such differences between NTG and POAG groups.

\section{DISCUSSION}

The present study was done with the aim to find out any differences in cognitive status using the MMSE scoring system with respect to age and sex-matched subjects among the three groups (controls, NTG, and POAG). The relationship between cognitive and visual decline has been an arguable topic nowadays. The progress of dementia determined by lower MMSE scores depends on its pathogenesis while as we know, glaucoma is also a neurodegenerative disease.
Table 1: Comparison of the mean CCT, RNFL, and MMSE among the controls, NTG, and POAG groups

\begin{tabular}{lllll}
\hline Parameter & $\begin{array}{l}\text { Controls } \\
(\mathbf{n = 2 0})\end{array}$ & $\begin{array}{l}\text { NTG } \\
(\mathbf{n = 2 0 )}\end{array}$ & $\begin{array}{l}\text { POAG } \\
(\mathbf{n = 2 0 )}\end{array}$ & p value \\
\hline CCT & $556.9 \pm 22.4$ & $521.0 \pm 25.8$ & $535.6 \pm 28.7$ & $<0.00^{*}$ \\
RNFL & $90.8 \pm 2.4$ & $76.62 \pm 7.8$ & $84.6 \pm 6.2$ & $<0.00^{*}$ \\
MMSE & $28.9 \pm 0.9$ & $25.2 \pm 3.8$ & $24.1 \pm 2.4$ & $<0.00^{*}$ \\
\hline
\end{tabular}

CCT: Central corneal thickness, RNFL: Retinal nerve fiber layer,

MMSE: Mini-mental state examination. ${ }^{\times}$Significant value. NTG: Normal-tension glaucoma, POAG: Primary open-angle glaucoma

In a previous study, the incidence of glaucoma was seen to be $26 \%$ in Alzheimer's Disease while only 5\% in controls [8]. Later in another study conducted by Tamura et al., glaucoma was seen prevalent in $24 \%$ of Alzheimer's patients. The study proposed that the lower ocular perfusion pressure leads to glaucomatous optic neuropathy which is seen in the early stages of Alzheimer's disease [9]. Yochim et al. concluded cognitive dysfunction in glaucoma, with number amounting to $44 \%$ [10]. Hagerman et al. also found that $32 \%$ of patients with low vision had impaired cognitive function [11].

MMSE score is used to evaluate the cognitive function of a person and screening of dementia with 30 being the maximum score. More accurate and comprehensive assessment could have been done by Montreal cognitive assessment (MoCA), where attention, language skills, and visuospatial processing were included [12]. In our study, we confined our three groups (controls, NTG, and POAG) only to the MMSE scoring system, since the educational level was poor, we could not include MoCA test. In our study, significant statistical differences were observed among the three groups. This is in accordance with the findings reported by Jefferis et al. [13]. The mean thickness of RNFL and CCT values in both the glaucoma groups were thinner as compared to controls. The results were consistent with the previous study [14].

Researchers have shown that glaucoma has few similar pathogenetic causes as that of Antithyroid drugs (ATD), which is the most common cause of dementia when both are related to aging. Recently, Yoneda et al. depicted that beta-amyloid and tau (neurofibrillary tangles) found in ATD pathology, do play important roles in glaucoma pathology. They also observed lower levels of beta-amyloid and a higher level of tau in the vitreous of glaucoma patients when compared with control group. In addition, it was found that beta-amyloid accumulates in the RGCs of rats with glaucoma induced for experimental purposes [15-18]. Thus, the study brought to us the hypothesis that lower cognitive scores were expected in glaucoma patients.

\section{Limitation of the study}

- Sample size (60) was relatively low

- Although one of the subjects in this study had good visual acuity, we could not confirm the effect of visual function on the MMSE scores

- Poor educational status forbade us to perform more sensitive tests to evaluate the cognitive status of the patients, such as the MoCA.

\section{CONCLUSION}

Since glaucoma and the dementia group of diseases possess same neurodegenerative pathogenesis affecting cognitive impairment, our findings support our hypothesis. Thus, it becomes necessary for an ophthalmologist to evaluate the cognitive status of the glaucoma patients to prevent early progression of dementia in the patients.

\section{AUTHOR'S CONTRIBUTIONS}

The authors have equally contributed to the project and highly grateful to the Department of Physiology for providing required facilities.

\section{CONFLICTS OF INTEREST}

The authors declared that they have no conflicts of interest. 


\section{REFERENCES}

1. Banerjee A, Khurana I. Altered autonomic balance in normal tension glaucoma. Asian J Pharm Clin Res 2017;10:175-7.

2. Ferri CP, Prince M, Brayne C, Brodaty H, Fratiglioni L, Ganguli M, et al. Global prevalence of dementia: A Delphi consensus study. Lancet 2005;366:2112-7.

3. Mandas A, Mereu RM, Catte O, Saba A, Serchisu L, Costaggiu D, et al. Cognitive impairment and age-related vision disorders: Their possible relationship and the evaluation of the use of aspirin and statins in a 65 years-and-over Sardinian population. Front Aging Neurosci 2014;6:309.

4. Rocchi A, Pellegrini S, Siciliano G, Murri L. Causative and susceptibility genes for Alzheimer's disease: A review. Brain Res Bull 2003;61:1-24.

5. Lin IC, Wang YH, Wang TJ, Wang IJ, Shen YD, Chi NF, et al. Glaucoma, Alzheimer's disease, and Parkinson's disease: An 8-year population-based follow-up study. PLoS One 2014;9:e108938.

6. Güngen C, Ertan T, Eker E, Yasar R, Engin F. Reliability and validity of the standardized mini mental state examination in the diagnosis of mild dementia in Turkish population. Turk Psikiyatr Derg 2002;13:273-81.

7. Valenti DA. Alzheimer's disease and glaucoma: Imaging the biomarkers of neurodegenerative disease. Int J Alzheimers Dis 2010;2010:793931.

8. Kessing LV, Lopez AG, Andersen PK, Kessing SV. No increased risk of developing Alzheimer disease in patients with glaucoma. J Glaucoma 2007; $16: 47-51$

9. Tamura H, Kawakami H, Kanamoto T, Kato T, Yokoyama T, Sasaki K, et al. High frequency of open-angle glaucoma in Japanese patients with Alzheimer's disease. J Neurol Sci 2006;246:79-83.

10. Yochim BP, Mueller AE, Kane KD, Kahook MY. Prevalence of cognitive impairment, depression, and anxiety symptoms among older adults with glaucoma. J Glaucoma 2012;21:250-4.

11. Hagerman KE, Taussig MJ, Coalter JD, Jay WM. Low-vision rehabilitation in patients with visual and cognitive impairment. Vis Impair Res 2007;9:19-22.

12. Mok GS, Wu YY, Lu KM, Wu J, Chen LK, Wu TH. Evaluation of the screening power of cognitive abilities screening instrument for probable Alzheimer's disease using voxel-based morphometry. Clin Imaging 2012;36:46-53.

13. Jefferis JM, Taylor JP, Collerton J, Jagger C, Kingston A, Davies K, et al. The association between diagnosed glaucoma and cataract and cognitive performance in very old people: Cross-sectional findings from the Newcastle 85+ study. Ophthalmic Epidemiol 2013;20:82-8.

14. Nouri-Mahdavi K, Nowroozizadeh S, Nassiri N, Cirineo N, Knipping S, Giaconi J, et al. Macular ganglion cell/inner plexiform layer measurements by spectral domain optical coherence tomography for detection of early glaucoma and comparison to retinal nerve fiber layer measurements. Am J Ophthalmol 2013;156:1297-307.e2.

15. Yoneda S, Hara H, Hirata A, Fukushima M, Inomata Y, Tanihara H. Vitreous fluid levels of beta-amyloid((1-42)) and tau in patients with retinal diseases. Jpn J Ophthalmol 2005;49:106-8.

16. Engelborghs S, De Vreese K, Van de Casteele T, Vanderstichele $H$, Van Everbroeck B, Cras P, et al. Diagnostic performance of a CSFbiomarker panel in autopsy-confirmed dementia. Neurobiol Aging 2008;29:1143-59.

17. Kaur K, Kaur R, Kaur M. Recent advances in Alzheimer's disease: Causes and treatment. Int J Pharm PharmSci 2016;8:8-15.

18. El Dayem SM, Metwally FD, Ahmed HH, Foda FM, Shalby AB, Zaazaa AM. Perspective in the treatment of Alzheimer's disease: Preclinical study. Int J Pharm PharmSci 2014;6:482-6. 